\title{
Estimates of anelastic dissipation in the Earth's torsional modes $(*)$
}

\author{
M. Caputo (**) \\ Ricevuto il 13 Gennaio 1966
}

Sumary. - The decay of the amplitude of the free modes of the Earth is a potential source of information on the mechanism of dissipation of the elastic energy of the Earth.

However there are serious difficulties in the interpretation of the decay; they are caused by several facts. One is the limited length of the significant part of the record which prohibits to identify the splitting of all the modes caused by the Earth rotation and also to follow the pattern in its rotation; another reason is the coupling between modes, caused by the inhomogenities and the flattening of the Earth, which can transfer energy from one mode to another. The results available are therefore very few and of poor accuracy.

In order to seek new information on the mechanism of dissipation of the elastic energy we solve a generalized form of the equation of elastodynamic in which we have introduced some unspecified operators to represent the dissipation of the elastic energy. By confronting these operators with the observations we would hope to find informations on the mechanism of dissipation. Unfortunately the laws of variation of $Q$ with frequency, found by various authors who were using different observations, are not in agreement and are very uncertain. Therefore we can only estimate the average values of the parameters of the supposed mechanisms of dissipation.

We analyze also the dissipation of energy due to viscous frictions at the core mantle boundary. This dissipation would be negligible even for viscosities of the core up to $10^{10}$ poise.

(*) This paper was presented at the $46^{\text {th }}$ Annual Meeting of the American Geophysical Union, Washington D. C., April 1965, and also at the Second International Symposium on Geophysical Theory and Computers, Rehovot, June, 1965.

(**) Department of Geophysics University of British Columbia Canada. 
Riassunto. - L'attenuazione delle oscillazioni libere della Terra è una sorgente d'informazione riguardo al meccanismo della dissipazione dell'energia elastica della Terra.

Purtroppo vi sono serie difficoltà, nell'interpretazione del decadimento delle oscillazioni, che hamno diverse origini. Cna è la limitata lunghezza della parte interessante delle registrazioni dei terremoti che impedisce la identificazione dello sdoppiamento delle linee spettrali causato dalla rotazione della Terra ed inoltre impedisce di seguire la rotazione di questa strutture di onde. Un'altra origine è l'accoppiamento dei modi di oscillazione causato dalle inomogeneità e dallo schiacciamento terrestre per cui vi è passaggio di energia da un modo all'altro. I risultati disponibili sono perciò pochi e di scarsa precisione.

Allo scopo di ottenere nuove informazioni riguardo al meccanismo di dissipazione dell'energia elastica, in questa nota, risolvo una forma generalizzata delle equazioni dell'elastodinamica in cui ho introdotto alcuni operatori che rappresemtano una vasta classe di modi di dissipazione dell'energia elastica. Dal confronto di questi operatori coi risultati delle osservazioni si possono ottenere nuove informazioni sul meccanismo di dissipazione dell'energia.

Sfortunatamente le leggi di variazione di $Q$ con la frequenza, trovate da vari autori che usano diverse osservazioni, non sono in accordo fra loro e sono molto incerte. E perciò difficile stabilire la legge di variazione di $Q$ con la profondita ed ho potuto solo stabilire dei valori medi per i parametri degli ipotetici meccanismi di dissipazione.

Ho altresi analizzato la dissipatione dell'energia causata dall'attrito viscoso al contorno fra il nucleo ed il mantello della Terra. Questa dissipa. zione risulta trascurabile anche per viscosità di $10^{10}$ poise.

\section{LIST OF SYMBOLLS}

$$
\begin{aligned}
& d=a(r) \quad \text { density } \\
& \sigma_{i}=\nu_{i j} \frac{\partial i}{\partial t^{j}}=f_{i}(j) \frac{\partial^{i}}{\partial t^{j}} . \quad \theta_{i}=\therefore \mu^{j} \\
& \therefore=\frac{\partial}{\partial r} \partial_{i}, \quad \partial_{i}==\frac{\partial}{\partial i} \theta_{i}
\end{aligned}
$$

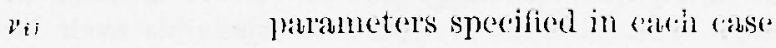

$$
\begin{aligned}
& P_{n}(\cos \theta) \quad \text { Texendre polynomials } \\
& \left.P_{n}(k) \cos \theta\right)=\sin ^{k} \theta \frac{d P^{k} P_{n}(x)}{\lambda x^{k}} \\
& Q^{-1} \quad \text { speceife: dissipation function } \\
& r, \forall, q \quad \text { polar coordinates } \\
& s\left(s_{1}, s_{2}, s_{3}\right) \quad \text {, displacement veretor and its Laplare transform in polat } \\
& S\left(S_{1}, S_{2}, S_{3}\right) \quad \text { coordinates }
\end{aligned}
$$


$T_{o}$

unperturbed period of torsional oscillation

$V_{o}$ gravitational potential

$V-V_{0} \quad$ perturbation of the gravitational potential

$w=$ coefficient of viscous friction at the core-mantle boundary

$\eta \quad$ - viscosity of the Maxwellian model

$\eta^{\prime}=$ viscosity of the Kelvin-Voigt model

$\lambda=\lambda(r), \mu=\mu(r)$ elastic parameters.

\section{TNTRODUCTION.}

In seeking improvements in the knowledge of the distribution of the density, elastic parameters, and parameters describing the energy dissipation in the Earth, there has recently been great emphasis on the observation and analyses of the free oscillations of the Earth and of their attenuation. The dissipation of energy of the free modes of the Earth is of interest, mainly if it is determined as a function of depth, because it provides a parameter from which we can gain additional information concerning the state of the constituents of the mantle.

Various causes of the dissipation of these modes have already been considered, such as the viscous and the electromagnetic interactions of core and mantle and the effect of the steady toroidal field on the lower boundary of the mantle (MacDonald and Ness, 1961; Kraut, 1965).

For the treatment of the viscous interaction, MacDonald and Ness considered the boundary as a flat plate, an approximation which is justified because the boundary layer thickness results of $10 \mathrm{~km}$ at most, even for the lowest periods and for core viscosities up to $10^{9}$ poises.

They gave the dissipation for viscosities of $10^{14}, 10^{4}$ and $10^{-6}$ poises for the torsional modes ${ }_{o} T_{9,}, T_{6},{ }_{1} T_{2}$ and for two earth models.

Values of the $Q$ of the torsional modes (Anderson and Archambeau, 1964, Mar'ussi, 1965) became available after the paper by MacIonald and Ness, and we thought it of interest to compute the viscosity of the core which would cause the observed dissipation.

This is done here for two simple Earth models consisting of a solid shell and a fluid core whose free torsional periods, for the low modes, are nearly the same as for the actual Earth and whose displacements at the core boundary are about $55 \%$ of those at the outer surface.

We find a simple formula which gives the $Q$ as a function of the viscosity and the period. In both models we find that the value of 
the riscosity which would cause the observed $Q$ should be of the order of $10^{11}$ poises; this seems too high.

Since the cause of the observed dissipation is not a riscous friction at the core-mantle boundary nor the interation of the magnetic and clastic fields, other causes of dissipation have to be considered.

Viscosities of about $10^{21}$ and $10^{22}$ poises of the upper part of the mantle as a Maxwellian solid can be estimated from the postglacial rebounds at Lake Bonneville (Crittenden 1963) and in Fennoscandia (Katriainen 1953), respectively. From these values, aceording to Knopoff and Maclonald (1958), the higher period limits, for a specific dissipation function independent of frequency, result of one and 10 years, respectively; therefore, if the constituents of the lower part of the mantle behave as those of the upper part, the dissipation of energy in the Farth's free modes should not depend on the viscosity of the mantle. The independence, however, of $Q$ from frequency is probably valid for some "anges of the temperature and pressure, and since composition phases, pressure, temperature of the lower part of the mantle are quite different from those of the erust, probably the lower limits of one or 10 years for the frequency independent dissipation function are not valid for the mantle. As a matter of fact, some author's have estimated frequency dependent $Q$ 's from propagation of Love and Reyleigh waves (Press, 1964) or from stress waves (Lomnitz, 1962).

A research made by Ben Menahem and Toksoz (1963) shows the Q frequency dependence of Love and Reyleigh waves in the range between 25 and 300 seconds; the specific dissipation results ahnost linearly dependent on frequency in that interval.

In another collection of data by Andersen and Archambeau (1964), the Q's of Love waves in the range between 20 and 600 seconds result quite scattered and there is no evidence of any relations with frequency; the $Q$ 's of Love waves indicate the probable presence of a minimum between 70 and 200 seconds and are consistently smaller than the $Q$ of Reyleigh waves (which involve also compression); the Q's of torsional modes instead result steadily increasing with the period in the range between 10 and 43 minutes, but the data in this case are very few. Andersen and Archambean (1964) obtained from these date some Earth models for $Q$, where $Q$ depends on depth, but, as they mention, the models are only tentative; better data in quality and quantity are needed.

Slichter et al. $(1964,1965)$ computed the Q's of the spheroidal modes which followed the 1964 dlaskan quake and which were observed at 
UCILA with the LaCoste and Rombergr tidal meters nos. -1 and 7 and also the $Q$ 's of the UCISA observations of the 1960 Chilean quake; they are reported in the figure, with the scatter of data from which they have been obtained. Even taking into aceount instrumental errors, these data show a trend and suggest that the Q's of these modes also increase with the period.

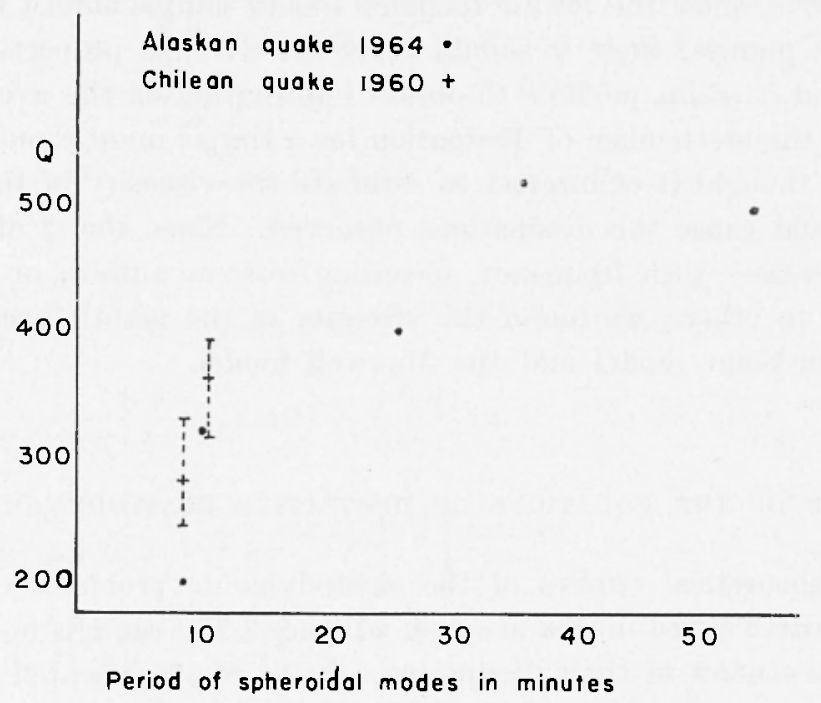

In a recent papere on the $y$ 's of the torsional modes recorded at Trieste, Marussi (1965) reported instend a $Q$ incleasing with frequency in the range between 2 and about 9 minutes; the $O$ then has a sudden drop and increases again at a period of about 20 minutes. We believe that this may be caused by the seiches of the near Gulf of Trieste and northern part of the Acliatic Sea. This is indicated also by Marussi but eamnot be definitely established until further research is done on these seiches.

According to Ness and MacDonald (1961), the low order torsional modes (i. e., periods of 15 minutes or more) have more or less constant energy throughout the mantle; these oscillations sample uniformly the entire mantle, and they are almost the same for the non-homogeneous and the homogeneous mantle. For the mode of 15.4 minutes the lower part of the mantle is less important since the energy distribution begins to drop rapidly at a depth of about $1500 \mathrm{~km}$. At periocls of 8 minutes the drop begins at $700 \mathrm{~km}$. Therefore, since diflerent wavelengths go through different layers of the mantle, and since $Q$ must be sensitive 
to changes of temperature, pressure, phase, and composition which are not exactly known and would eover any frequenty dependent law, at this stage of the research in this field and with the data available, it would be hard to attempt any study of frequency dependent $Q$ from the free modes of the Earth.

Although the data available does not allow the investigation mentioned above, since the lowest torsional modes sample almost uniformly the entire mantle, their $Q$ should represent average properties of the mantle and it seems possible to obtain information on the arerage properties of the mechanism of dissipation for a simple mantle model. We, therefore, thought it of interest to estimate the viscosity of the mantle which would cause the dissipations observed. Since the $Q$ of the free modes increases with frequency according to some authors or decreases according to others, we found the viscosity of the mantle according to the Kelvin-Voigt model and the Maxwell model.

\section{SOLUTIONS OF THIF EQUATIONS OF DISSIPATIVE ELASTODYNANITS.}

The theoretical studies of the elastodynamic problems connected with the earth's free modes are well advanced; the same is not true for theoretical studies of their dissipation. None of the research done has treated theoretically the combined dissipative elastodynamic problem from a general point of view which is needed for the discussion of some of the problems mentioned in the introduction.

In this direction, we shall give a general solution of the equation of motion of a dissipative elastic gravitating non-homogeneous isotropic: field whose elastic parameter and density are functions of the distance from a point which will be assumed as the origin of a system of spherieal coordinates and whose mechanism of dissipation is generic and could later be specified as that of Maxwellian viscosity or that of solid friction or of other kinds of dissipation. (For a description of the various models of losses see Knopoft [196t]).

For this purpose let us consider the following system of partial differential equations:

$$
\begin{gathered}
F_{1} \equiv o_{1} \frac{\partial \Delta}{\partial r}+\frac{2 o_{2}}{r \sin \vartheta}\left(\frac{\partial \omega_{\theta} g}{\partial \varphi}-\frac{\partial \omega_{\varphi} \sin \dot{v}}{\partial \theta}\right)+o_{1} \Delta+2 o_{2}\left(\frac{\partial u}{\partial r}-\Delta\right)+ \\
+o_{3} u-d \frac{\partial V_{0}}{\partial r} \Delta+\frac{d \partial\left(V-V_{o}\right)}{\partial r}+a \frac{\partial}{\partial r}\left(u \frac{\partial V_{o}}{\partial r}\right)=0
\end{gathered}
$$




$$
\begin{aligned}
& F_{2} \equiv\left\{o_{1} \frac{\partial A}{\partial \theta}+2 o_{2}\left(\frac{\partial r \omega_{\varphi}}{\partial r}-\frac{1}{\sin \vartheta} \frac{\partial(\omega r}{\partial \varphi}\right)\right\} r^{-1}+\delta_{2}\left(\frac{1}{r} \frac{\partial u}{\partial \theta}-\frac{v}{r}+\frac{\partial v}{\partial r}\right)+ \\
& +o_{3} v+\frac{\lambda}{r} \frac{\partial\left(V-V_{o}\right)}{\partial \theta}: \frac{\lambda}{r} \frac{\partial}{\partial \vartheta}\left(\because \frac{\partial V_{o}}{\partial r}\right)=0
\end{aligned}
$$

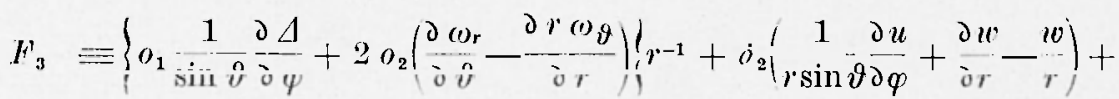

$$
\begin{aligned}
& +o_{3} w+\frac{a}{r \sin \vartheta} \frac{\partial\left(V-V_{o}\right)}{\partial \varphi}+\frac{d}{r \sin \vartheta \partial \partial}\left(u \frac{\partial V_{o}}{\partial r}\right)=0 \\
& H_{4}=A^{2}\left(V-V_{0}\right)-4 \pi G\left(d \Delta+u \frac{\partial d}{\partial r}\right)=0 \text {. } \\
& \Delta=\frac{1}{r^{2}} \frac{\partial}{\partial r}\left(r^{2} s_{1}\right)+\frac{1}{r \sin \vartheta} \frac{\partial}{\partial \eta}\left(s_{2} \sin \vartheta\right)+\frac{1}{r \sin \vartheta} \frac{\lambda \varrho}{\partial \varphi} \\
& \omega_{r}=\frac{1}{2 r \sin \vartheta}\left\{\frac{\partial}{\partial \tau}\left(s_{3} \sin \vartheta ;-\frac{\partial s_{2}}{\partial \varphi}\right\}\right. \\
& \omega_{\vartheta}=\frac{1}{2 r \sin \theta}\left\{\frac{\partial s}{\partial q}-\frac{\partial}{\partial r}\left(r s_{3} \sin \dot{\theta}\right)\right\} \\
& \omega_{\varphi}=\frac{1}{2 r}\left\{\frac{\partial\left(r s_{2}\right)}{\partial r}-\frac{\partial s_{1}}{\partial \vartheta}\right\} \\
& o_{i}==\nu_{i} \frac{\partial j}{\partial t^{j}} \\
& \dot{d}_{i} \equiv \frac{\partial v_{i j}}{\partial r} \frac{\partial j}{\partial t^{j}} .
\end{aligned}
$$

It is immediately recognized that if

$$
o_{1}=v_{10} \quad o_{2}=v_{20} \quad o_{3}=-v_{32} \frac{\partial^{2}}{\partial t^{2}}
$$

then [1] are the equations which govern the motion of an elastic field of elastic parameters $\lambda=v_{10}-2 v_{20}, \mu=v_{20}$ density $-v_{32}$ and gravitational potential $V$. The perturbation $V-V_{o}$ is that of the gravitational potential and arises from the perturbation of the density field and from the attraction of the density perturbation at the deformed interfaces.

It should be noted that in order to have the problem properly set, i. e. to have all the equations governing the motion of the field, we have to associate to [1] the equations expressing the boundary and the initial 
conditions. Ilowever, we will give here a solution of [1]; the boundary and initial conditions will be satisfied later by means of the arbitary constants of the solution. To find a solution of equations [1] let us now write the following equations which are a consequence of [1].

$$
\begin{aligned}
& \int_{w} H_{1} X_{n}^{k} d(1)=0 \\
& \int_{i \omega}\left|F_{3} \frac{1}{\sin \vartheta} \frac{\partial Y_{n}^{k}}{\partial \varphi}+F_{2}^{\prime} \frac{\partial Y_{n}^{k}}{\partial \vartheta}\right| d(1)=0 \\
& \int_{\dot{w}}\left|F_{2} \frac{1}{\sin \vartheta} \frac{\partial Y_{n}^{k}}{\partial \varphi}-F_{3} \frac{\partial Y_{n}^{k}}{\partial \vartheta}\right| d(\omega)=0 \\
& \int_{i} F_{d} Y_{n}^{k} d(\omega)=0 \\
& \int_{\dot{w}} L d \omega=\int_{0}^{2 \pi} d \varphi \int_{0}^{\pi} \sin \vartheta d \vartheta \int_{0}^{+\infty} L e^{i n t} d t . \\
& Y_{.}^{k}= \begin{cases}\left(\frac{2 n+1}{1 \pi}\right)^{1 / 2} P_{n}(\cos \vartheta) & \text { if } k=0 \\
\left.\mid \frac{2 n+1}{2 \pi} \frac{(n-k) !}{(n+k) !}\right]^{1 / 2} P_{n}^{(k)}(\cos \vartheta) \cos k \varphi & \text { if } k=1,2 \ldots n[-1] \\
\left|\frac{2 n+1}{2 \pi} \frac{(2 n-k) !}{k !}\right|^{1 / 2} P_{n}^{(k-n)}(\cos \vartheta) \cos (k-n) \varphi & \end{cases} \\
& \text { if } k=n+1, \ldots 2 n
\end{aligned}
$$

and set

$$
\begin{aligned}
& R_{1, n}=\int_{\dot{v}} s_{1} Y_{n}^{k} d(\omega) \\
& R_{2, n}=\int_{w}\left|s_{2} \frac{\partial Y_{u}^{\dagger}}{\partial \vartheta}+s_{j} \frac{\partial Y_{*}^{k}}{\partial \varphi} \frac{1}{\sin \hat{v}}\right| d \omega \\
& R_{3, n}=\int_{\dot{w}}\left[s_{2} \frac{1}{\sin \vartheta} \frac{\partial Y_{n}^{i}}{\partial \varphi}-s_{3} \frac{\partial Y_{v}^{n}}{\partial \vartheta}\right] d \omega \\
& \left(P_{n}-P_{o, n}\right)=\dot{\int}\left(V-V_{0}\right) Y_{n}^{k} d(1) .
\end{aligned}
$$


Performing the integrations in [3], we see that if the vectors $R_{n}\left(R_{1, n}, R_{2, n}, R_{3, n}\right)$ and $R_{t, n}$ are solutions of the obtained system [6],

$$
\begin{aligned}
& \frac{d}{d r}\left|O_{1} \bar{\nabla}\right|+2 O_{2} \frac{d \nabla}{d r}-O_{2}\left|\frac{R_{1}}{r} n(n+1)-\frac{1}{r^{2}} \frac{d\left(r R_{2}\right)}{d r} n(n+1)\right|+ \\
& +2 \dot{O}_{1} \frac{d R_{1}}{d r}-d \frac{d P_{0}}{d r} \nabla+\frac{d\left(P-P_{0}\right)}{d r}+d \frac{d}{d r}\left(R_{1} \frac{d P_{0}}{d r}\right)+ \\
& +O_{3} R_{1}=0 \\
& r-1\left\{O_{1} \overline{\bar{\nabla}}-O_{2}\left[\frac{d R_{1}}{a r}-\frac{d^{2}\left(r R_{2}\right)}{d r}\right]+O_{2} r\left[\frac{R_{1}}{r}+r \frac{d R_{2} / r}{a r}\right]\right\}+ \\
& +\frac{d}{r}\left(P-P_{o}\right)+d \frac{R_{1}}{r} \frac{d P_{o}}{d r}+O_{3} R_{2}=0 \\
& \frac{1}{r} \frac{d}{d r} \mid r^{2} \frac{d\left(P-P_{0}\right)}{a r}-1 \pi d G \bar{\nabla}+\frac{n(n+1) P-P_{0}}{r}+\pi G \frac{d d}{r} R_{1}=0 \\
& O_{2}\left[\frac{1}{r} \frac{d^{2} r R_{3}}{d r}-\frac{n(n+1)}{r} R_{3}\right]+\dot{O}_{2} r \frac{d R_{3} / r}{d r}+O_{3} R_{3}=0 \\
& \overline{\bar{\nabla}} \equiv-\frac{n(n+1)}{r} n_{0}+\frac{1}{r} \frac{d}{d r}\left(r^{2} R_{1}\right) \text {. }
\end{aligned}
$$

then they determine the Fourier coordinates of the Laplace (or Fourier) transforms $S\left(S_{1}, S_{2}, S_{3}\right)$ and $P-P_{0}$ of the vector $s\left(s_{1}, s_{2}, s_{3}\right)$ and of the perturbation $\left(V-V_{0}\right)$. In fact from [5] it can be shown that, extending the procedure used by Caputo (1963), we can formally write.

$$
\begin{aligned}
& S_{1}=\sum_{n} \sum_{k} R_{1} \vartheta_{n}^{k} \\
& S^{\prime}=\sum_{n} \sum_{k}\left(R_{2} \frac{\partial Y_{n}^{k}}{\partial \vartheta}+R_{3} \frac{1}{\sin \vartheta} \frac{\partial Y_{n}^{k}}{\partial \varphi}\right) \\
& S_{3}=\sum_{k} \sum_{k}\left(R_{2} \frac{1}{\sin \vartheta} \frac{\partial Y_{n}^{k}}{\partial \varphi}-R_{3} \frac{\partial Y_{i}^{\prime}}{\partial \vartheta}\right) \\
& \Psi=\Psi_{o}+\sum_{n} \sum_{k}\left(P-P_{o}\right) Y_{n}^{i} .
\end{aligned}
$$

It is interesting to note that in equations [6], as in those resulting in case of the absence of dissipation (Caputo 1963), the $R_{3}$ component is governed by a single equation (the last of the set) not involving $o_{1}$, $R_{1}, R_{2}$, nor the gravitational potential.

A particularly interesting solution of [1] is that with $R_{1}=: R_{2}$, $r-V_{o}=0, I^{k}=P_{n}^{k}$, which represents motions along parallels with 
rotational symmetry; the periods of the free modes of this type are obtained from the last equation of the set. If, in the case

$$
\begin{gathered}
R_{1}=R_{2}=V=V_{o}=0, Y_{i i}^{k}=P_{n}^{k} \text { we set } o_{2}=\nu_{20}+\nu_{21} \frac{\partial}{\partial t} \\
o_{3}=-v_{32} \frac{\partial^{2}}{\partial t^{2}}
\end{gathered}
$$

then we obtain the equation governing the torsional motions of an elastic field in which the mechanism of dissipation is of the Kelvin-Voigt type. If we set $o_{2}=v_{21}, o_{3}=-v_{31} \frac{\partial}{\partial t}-v_{32} \frac{\partial^{2}}{\partial t^{2}}$ then we obtain the equation of the field in which the mechanism of dissipations is of the Maxwell type.

When considering the elastic field to be a spherical shell of radiae $r_{1}$ and $r_{2}$, the boundary conditions, in the case of axial symmetry, which is of interest in the problems on the free modes, are

$$
\begin{aligned}
& P_{1,1}=\left[\lambda \Delta+2 \mu \frac{\partial s_{1}}{\partial r}\right]_{\substack{r=r_{1} \\
r=r_{2}}}=-\left|\begin{array}{l}
f_{1}(\vartheta, t) \\
g_{1}(\vartheta, t)
\end{array}\right|_{\substack{r=r_{1} \\
r=r_{2}}} \\
& P_{1,2}=\left[\mu\left(r \frac{\partial s_{2} / r}{\partial r}+\frac{\partial s_{1}}{r \partial \vartheta}\right)\right]_{\substack{r=r_{1} \\
r=r_{2}}}=-\left[\begin{array}{l}
f_{2}(\vartheta, t) \\
g_{2}(\vartheta, t)
\end{array}\right]_{\substack{r=r_{1} \\
r=r_{2}}}
\end{aligned}
$$

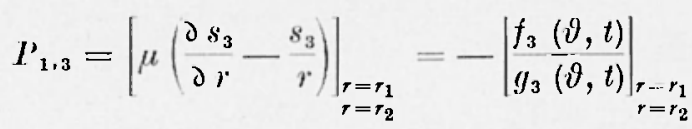

where $P_{i j}$ are the components of the surface stress and $f_{i}, y_{i}$ the romponents of the forces applied there. To satisfy conditions [8], we can apply the method of the integral properties; let us multiply both members of the first of [8] by $e^{-p t} P_{n}(\cos \vartheta) \sin \vartheta$, the others by $e^{-p t} \frac{\partial P_{n}}{\partial \vartheta} \sin \vartheta$ and integrate, we have

$$
\left[\frac{\lambda}{r^{2}}\left(\frac{\partial r^{2} R_{1, n}}{\partial r}-r R_{2, n}\right)-2 \sum_{\mu i} \frac{\partial R_{1, n}}{\partial r}\right]_{\substack{r=r_{2} \\
r=r_{1}}}=-\int_{i}^{\pi} P_{n} \sin \vartheta \int_{0}^{\infty}\left(\begin{array}{l}
f_{1} \mid \\
g_{1} \mid
\end{array} e^{-p \ell} d t d \vartheta\right.
$$


$\left|\mu\left(r \frac{\partial R_{-,-w}}{\partial r}+n(n+1) \frac{1}{r} R_{1, n}\right)\right|_{\substack{r=r_{2} \\ r=r_{1}}}=-\int_{0}^{\pi} \frac{\partial P_{n}}{\partial \vartheta} \sin \vartheta \int_{0}^{\infty}\left(\begin{array}{l}f_{2} \\ g_{2}\end{array}\right) e^{-p t} d t d \theta$

$\left|\mu\left(\frac{\partial R_{3, n}}{\partial r}-\frac{1}{r} R_{3, n}\right)\right|_{\substack{r-r_{2} \\ r=r_{1}}}=-\int_{0}^{\tau} \frac{\partial P_{n}}{\partial \vartheta} \sin \vartheta \int_{0}^{\infty}\left(\begin{array}{l}f_{3} \\ g_{3}\end{array}\right) e^{-p t} d t d \vartheta$.

EIRTH MODELS.

For mathematical convenience in the discussion of the dissipation in the Earth's free torsional modes, we assume for the mantle a model with constant density, $o_{2}=r^{2} \nu_{2 i} \frac{\partial_{i}}{\partial t_{i}}, o_{3}=\nu_{3 i} \frac{\partial^{i}}{\partial t^{i}}$ and rigridity $\mu=y_{20}$ with:

$d \quad=v_{32}=4.47, \quad r_{2}=6.371 \cdot 10^{8}$,

$r_{1}=3.437 \cdot 10^{8}, \quad v_{20}=3.095 \cdot 10^{-6}\left(\right.$ all the other $y_{j i}=0$ )

whose free torsional periods, in the low frequencies absence of dissipation, (all the $v_{i j}=o$ except $\nu_{32}, v_{20}$ ) are different from those observed for few parts percent. $R_{3, n}$ then are given by (Caputo 1961)

$$
R_{3, n}=C_{1, n} r^{m_{1}}+C_{2, n} r^{m_{2}}
$$

where $m_{1}$ and $m_{2}$ are

$$
m_{1,2}=-\frac{3}{2} \pm\left\{t+\frac{v_{3 i} p^{i}}{v_{2 i} p^{i}}+\left(n+\frac{1}{2}\right)^{2}\right\}^{1 / 2}
$$

If we chose the mantle model with $o_{2}=v_{2 i} \frac{\partial i}{\partial i^{i}}, o_{3}=-r^{-2} \nu_{3 i} \frac{\partial i}{\partial t^{i}}$

$$
\begin{aligned}
& d=v_{32} r^{-2} \quad, \quad \mu=v_{20} \\
& r_{1}=3.437 \times 10^{8}, \quad r_{2}=6.371 \times 10^{9} \\
& v_{32}=1.1145 \times 10^{18}, \quad v_{20}=1.74 \times 10^{12}
\end{aligned}
$$

whose free torsional modes, in case of absence of dissipation, also have periods which differ from the observed ones for few parts percent, then the solution $R_{3, n}$ is as in [11] with

$$
m_{1,2}=-\frac{1}{2} \pm\left\{\frac{\nu_{3 i} p^{i}}{p_{2} p^{i}}+\left(n+\frac{1}{2}\right)^{2}\right\}^{1 / 2}
$$


The periods of the free torsional modes of the two models are obtained setting $f_{i}=y_{i}=0$ in conditions [9]

$$
\left|\begin{array}{ll}
\left(m_{2}-1\right) r_{2}^{m_{1}+1} & \left(m_{2}-1\right) r_{2}^{m_{2}+1} \\
\left(m_{1}-1\right) r_{1}^{m_{1}+1} & \left(m_{2}-1\right) r_{1}^{m_{2}+1}
\end{array}\right|=0
$$

The equation for the free periods is therefore for both models

$$
\left[\nu_{31}+\nu_{2 i}\left(n^{2}+n-2\right)\right] p^{i}=0 \text {. }
$$

In the free modes the values of $m_{1}$ and $m_{2}$ are for every $n$ and model [11].

$$
m_{1}=1, \quad m_{2}=-4
$$

and for model [13]

$$
m_{1}=1, \quad m_{2}=-2
$$

$s_{n}$ is for both models

$$
s_{n}=\frac{A_{n} r}{r_{2}} P_{n}^{1}(\cos \theta) e^{n t}
$$

where $A_{n}$ is the amplitude of the $n$ mode at the surface $r=r_{2}$. From [17], therefore, we see that the displacements at the core-mantle boundary of our models are about $55 \%$ of the displacements at the outer surface. It can also be seen that in the free modes of these two models there are no nodal surfaces. The models are therefore suitable for the study of the problem considered below, at least for the lowest frequencies.

\section{DINSIPATION DUE TO FRICTION AT TIIE CORE-MANTLE BOUNDARY.}

A mechanism of dissipation in the Earth's free modes is the friction at the core-mantle boundary. In this case the boundary condition equations are [9] with $f_{i}=0$

$$
g_{i}=\frac{r_{1}^{2} w}{r_{1}-r_{o}} \frac{\partial s_{i}}{\partial t}
$$

where $w$ is the coefficient of the viscous damping and $r_{1}-r_{o}$ the boundary layer. The last two of conditions [7] become after integration 


$$
\begin{aligned}
& \left(\frac{\partial R_{3}}{\partial r}-\frac{R_{3} \mid}{r / r=r_{2}}=0\right. \\
& / \mu\left(\frac{\partial R_{3}}{\partial r} \cdot{ }^{2}-r R_{3}\right)_{r=r_{1}}+p w r_{:}^{2} \frac{R_{3}}{r_{1}-r_{0}}=0 .
\end{aligned}
$$

The equation for the periods of the free torsional modes is therefore

$$
\begin{array}{cc}
\left(m_{1}-1\right) r_{2}^{m_{1}+1} & \left(m_{2}-1\right) r_{2}^{m_{2}+1} \\
\mu\left(m^{1}-1\right) r_{1}^{m_{1}+1}+p \frac{w r_{1}}{r_{1}-r_{0}} r^{m_{1}+1} & \mu\left(m_{2}-1\right) r^{m+1}+p \frac{w r_{1}}{r_{1}-r_{0} r_{1}^{m_{2}+1}}
\end{array}
$$

If we choose the mantle model [13] with $y_{2 i}=v_{3 i}=0$ except $v_{20}$, $\nu_{32}$, then the significant solutions of [20] are given by

$$
\begin{aligned}
& \left(\mu\left[\frac{9}{4}-p^{2} \frac{d_{o}}{\mu}-\left[n+\frac{1}{2}\right]^{2}\right]-\frac{3}{2} \frac{p w r_{1}}{r_{1}-r_{o}}\right)\left(1-a^{\left.-2 \mid p^{2} \frac{d_{0}}{\mu}+\left(n+\frac{1}{2}\right)^{2 /\left.\right|^{1 / 2}}\right)}+\right. \\
& +\left(\frac{d_{o}}{\mu} p^{2}+\left[n+\frac{1}{2}\right]^{2}\right)^{1 / 2} \frac{p v r_{1}}{r_{1}-r_{o}}\left(1+n^{-2}\left[p^{2} \frac{o}{\mu}+\left(n+\frac{1}{2}\right)^{\mu^{2 / 2}}\right]^{-12}\right)=0, \\
& \alpha \doteq \frac{r_{1}}{r_{2}} \text {. }
\end{aligned}
$$

To solve equation [21] let $p=p_{o}+\Delta p$ where $p_{o}$ is associated to the period for $w=0$ and therefore satisfies the equation [21] with $w=0$ and $\Delta p<10^{-2} p_{0}$. With these assumptions equation [21] can bo written

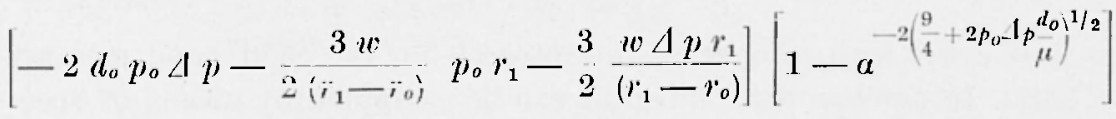

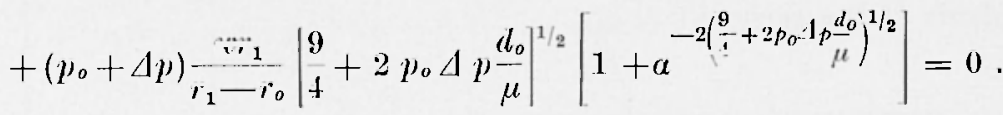

Since $\Delta p<p_{0} 10^{-2}$, for low frequencies and $w<10^{13} \quad(n \leqslant 20)$, the term $-\frac{3}{2} \frac{w r_{1}}{r_{1}-r_{0}}$ is negligible in respect to $-2 \mu_{o} p_{\circ}$, also the 
term $2 p_{o} \Delta p \frac{d_{0}}{\mu}$ for low frequencies is negligible in respect to $\frac{9}{4}$; therefore we can simplify equation $[22]$ to

$$
\Delta p^{2}+\frac{3\left(a^{3}-1\right) \mu\left(r_{1}-r_{o}\right)}{w r_{1}\left(a^{3}+1\right)} \Delta p+\frac{9}{2} \frac{\mu}{d_{o}\left(a^{3}+1\right)}=0
$$

$\Delta p$ is:

$$
\Delta p^{-1}=+\frac{2\left(r_{1}-r_{o}\right)\left(a^{3}-1\right) d_{o}}{3 r_{1} w}
$$

and the perturbed period

$$
T=T_{o}+\frac{9 r_{1}^{2} w^{2} T_{o}^{3}}{8 \pi^{2}\left(r_{1}-r_{o}\right)^{2} d_{o}^{2}\left(a^{3}-1\right)^{2}} .
$$

From the formula above it results that the perturbation of the periods would therefore be negligible even for $w=10^{13}$ poises.

In the case under consideration formula [17] is

$$
s_{n}=A_{n} \frac{r}{r_{2}} P_{n}^{1}(\cos \vartheta) e^{i p_{0} t} e^{i p t}
$$

and according to the definition of $Q$ we have for model [13]

$$
\ell=\frac{2 \pi E}{\Delta E}-\frac{\pi}{T \Delta p}-\frac{2 \pi\left(r_{1}-r_{o}\right)\left(1-a^{3}\right) d_{n}}{3 r_{1} w T} .
$$

The same results are valid for model [10].

In case that $Q$ has to be obtained from observations $\Delta p$ is given by

$$
\Delta p=\frac{1}{2} \Delta \ln \left|\begin{array}{c}
\left.\right|_{t_{2}} ^{t_{2}} d t \\
\frac{t_{1}}{t_{0}+\Delta} \\
\prod_{1}+s_{n}^{2} \partial t
\end{array}\right|
$$

as it is a asily seen computing the integrals on the right hand member of [24]. In practice these integrals can be computed by means of spectral analysis of the records.

ANALYSIS OF OBSERVATIONS.

Values of $Q$ of the free torsional modes have been obtained by several anthors. The values given by Anderson and Archambean (1964) 
are for periods between 10 and 20 minutes and increase from 200 to 400 , in agreement with rariation of $Q$ of the spheroidal modes (Slichter et al., 1964). The $Q$ of the shear waves for periods between 40 and 300 s also increases with the period (Press 1964a, 1964b).

Anderson and Archambeau have developed accordingly a technique for determining $Q$ as a function of depth; the values are 80 for the upper mantle, 2000 for the middle mantle, and 5000 (as a lower bound) for the lower mantle. Different results have been obtained by Marussi (1965) from the analysis of the observations of the Chilean quake male with the Trieste pendulum. He found values of $Q$ decreasing with an increasing period, for periods from 20 to 4 minutes, but from 4 to 2 minutes the $Q$ is almost constant. We shall discuss these difierent results separately.

Using the values of the dissipation obtained by Marussi (1965), it is seen that the viscosity of the core at the mantle boundary, in order to give a significant contribution to this dissipation, should have the values listed in Table I. The computation is made as follows.

The thickness of the boundary layer which reduces the amplitucle to 0.01 is

$$
\left(r_{1}-r_{0}\right)=0.84 \text { v } T^{\prime}
$$

By combining [24] and [24'] we obtain for w the values listed in the following table

'Table I.

\begin{tabular}{|c|c|c|c|c|}
\hline Jeriod & $\begin{array}{l}\text { Estimated specif. } \\
\text { dissip. due to } \\
\text { viscosity ai core- } \\
\text { mantle bommdary }\end{array}$ & $\begin{array}{l}\text { Total specif. } \\
\text { dissipation }\end{array}$ & $w$ & $\begin{array}{c}\text { 13oundary } \\
\text { layes } \\
r_{1}-r_{0}\end{array}$ \\
\hline $28^{\mathrm{m}}$ & $2.2 \cdot 10^{-3}$ & $220^{-1}$ & $2 \cdot 2 \cdot 10^{11}$ & $1.6 \cdot 10^{7}$ \\
\hline $10 \mathrm{~m}$ & $12 \cdot 10^{-3}$ & $280^{-1}$ & $1.8 \cdot 10^{11}$ & $0.9 \cdot 10^{7}$ \\
\hline $4^{\mathrm{m}}$ & - & $405^{-1}$ & - & $\ldots$ \\
\hline
\end{tabular}

Because of the assumptions on $Q$ the above values of 10 are 10 be considered upper limits. 


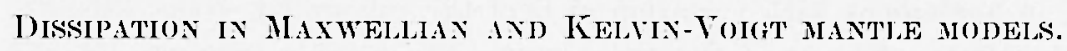

We have just seen that the observed dissipation of the Earth's modes camnot be caused by the core viscosity at the core-mantle boundary. Inother suggestion could be that the cause of dissipation is the viscosity of the mantle.

The values of $Q$ observed at Trieste suggest a Maxwellian model for the viscosity, while the values of the other observers suggest a viscosity according to the Kelvin-Voigt model.

In this section, we shall find an estimate of the viscosity of mantle according to the two models of viscosity; $\eta$ will be the viscosity of the Maxwellian model and $\eta^{1}$ that of the model of Kelvin-Voigt. The theory will be treated for the unified case of a Kelvin-Voigt-Maxwell model, and then the two cases will be discussed separately. The equation of motion for the torsional oscillation of model [11], in this case, can be written to the first order (i. e. neglecting the terms representing the interaction between the two mechanisms of the Kelvin- Toigt and Maxwell type of losses)

$\left(\mu_{o}+\eta^{1} p\right)\left(\frac{\partial^{2} R}{\partial r^{2}}+\frac{2 \partial R_{3}}{r}-\frac{\left.n(n+1) R_{3}\right)}{r^{2}}\right)-\left(\frac{\mu_{0} l_{0}}{\eta} p+l_{o} p^{2}\right) \frac{R_{3}}{r^{2}}=0$

[25] is obtained from [6] with

$$
o_{2}=\left(\mu_{0}+\eta^{1} \frac{\lambda}{i} i\right), \quad o_{3}=-\left(\frac{\mu_{0} d_{o}}{i j} p+l_{o} p^{\prime}\right) r^{-2}, \quad R_{1}=R_{2}=0 .
$$

We can proceed to integrate [25] as we did for [1] and obtain for model [13], with viscosities, independent of coordinates, the following values of $m_{1}$ and $m_{2}$

$$
m_{1,2}=-\frac{1}{2} \pm\left\{\frac{d_{0}}{\eta^{1} p+\mu_{0}} p^{2}+\frac{\mu_{0} l_{o}}{\eta\left(\eta^{1} p+\mu_{o}\right)} p+\left(n+\frac{1}{2}\right)^{2}\right\}^{1 / 2} .
$$

The periods of the torsional modes are governed by the equation

$$
p^{2}+\frac{\mu_{0}}{\eta} p+\frac{\mu_{0}+\eta^{1} p}{a_{0}}\left(n^{2}+n-2\right)=0
$$

which is analogous to [17] and is obtained assuming $f_{i}-y_{i}=0$ in the boundary conditions [7]. The corresponding values of $Q^{-1}$ for model [13] are therefore: 


$$
\begin{gathered}
q=\frac{\mu_{o} T}{\pi 2 \eta}+\frac{2 \pi \eta^{1}}{\mu_{o} T} \\
Q^{-1}=2.8 \cdot 10^{11} \frac{T_{o}}{\eta}+0.36 \cdot 10^{-11} \frac{\eta^{1}}{T_{o}} .
\end{gathered}
$$

From the values of $Q$ given by Anderson and Archambean (196-4), assuming $\frac{1}{\eta}=0$, we find for $\eta^{1}$ the following values

Table II. - KeIvix-VorgT MonsL.

\begin{tabular}{|c|c|c|}
\hline Period & Spec. Dissip. & $1^{1}$ \\
\hline $30^{\mathrm{m}}$ & $400^{-1}$ & $1.2 \cdot 10^{11}$ \\
$20 \mathrm{~m}$ & $300^{-1}$ & $1.1 \cdot 10^{11}$ \\
$10^{\mathrm{m}}$ & $200^{-1}$ & $0.8 \cdot 10^{11}$ \\
\hline
\end{tabular}

From the values of $Q$ observed at Trieste, assuming $\eta^{\prime}=0$ we find for $\eta$ the following values:

Table II. - Maxwel. Moder.

\begin{tabular}{|c|c|c|}
\hline Period & Spec. Dissip & $\eta$ \\
\hline & & \\
\hline $10^{\mathrm{m}}$ & $2800^{-1}$ & $+.8 \cdot 10^{17}$ \\
$4^{\mathrm{m}}$ & $405^{-1}$ & $6.7 \cdot 10^{17}$ \\
\hline
\end{tabular}

These values for the viscosity of the mantle should not be compared with the values obtained from the uplifts of Lake Bommeville $\left(2 \cdot 10^{21}\right.$ poise) or of Fennoscandia (2. 10: poise) because these phenomena are on a different time scale and they interest only the upper part of the mantle. Moreover, there are still some doubts on viscosities determined from these uplifts. In fact the major part of recovery took place before complete removal of the ice load and we know very little of the uplifts at the early stages of deglaciation; also we do not know what degree of isostasy was achieved during the glaciation. 
The value of the viscosity of the mantle found from the departure of the Earth's actual figure from that of hydrostatic equilibrium is not too significant. In fact that departure is not supposed to have developed during the last 10 million years of deceleration of the Earth's rotation, but it is probably a lag between the two figures, which has existed since longer.

Marussi mentioned that while the $Q^{\prime}$ s of the periods of the order of several minutes are not influenced by the seiches of the gulf of Trieste, or of the northern part of the Adriatic sea, the $Q^{\prime} \mathrm{s}$ of the lower frequencies are probably influenced by these seiches. We believe that these seiches could also give an explanation of the increasing $Q$ with the frequency found in Trieste. $\Lambda \mathrm{n}$ analysis of Trieste pendulum records during a period without large earthquakes (Zadro 1961) showed periods of $420,240,210,98,93,80,70$ minutes in agreement with the periods of the seiches of the Adriatic Sea directly computed from tide-ganges records (Caloi 1938, Polli 1958); but the analysis of Zadro as well as that of the mareograf records was made on a time series whose nyquist frequency was $2.5 \mathrm{cph}$; therefore, the periods which interest the discussion of the $Q$ obtained at Trieste could not be found.

We estimate that the Gulf of Trieste has seiches with periods of about $17,11,8.5,6.8,5.7, \ldots$ minutes, and the basin from the northern east coast to the west coast of the $\Lambda$ driatic Sea has seiches with periods of about $29,17,15,13,11.6, \ldots$ minutes, which cover the range of the low free torsional modes of the Earth. We, therefore, believe it possible that the variation of $Q$ with frequency found in Trieste is not in agreement with the variation of $Q$ found from other observations and with that of the shear waves because of perturbations caused by the above-mentioned seiches. A spectral analysis of tide-ganges and pendulums' records in Trieste, with higher nyquist frequency, would certainly throw light on this problem.

\section{ConClusion.}

The friction at the core-mantle boundary is found to be a mechanism of negligible importance in the dissipation of energy of the Earth's free modes. The results obtained here for the various viscosities of the mantle from the observed $Q$ of the torsional modes are only tentative. 
Using the same theory analogous analysis can also be made for the spheroidal modes. In both cases, when the knowledge of $Q$ will be more detailed, it will be possible to introduce in the theory more sophisticated multilayered models (and also more, sofisticated mechanisms of dissipation) and, since the energy of the different modes is stored in different layers of the Earth, the viscosities in these models could be varied with depth.

\section{BIBLIUGRAPII}

Alsop L. E., Sutton G. H., Ewixg M. E., Measurement of $Q$ for very long period free oscillations. "J. Geopl. Res.", 66, 2911-2915, (1961).

Anderson Don L. and ARCinambenu C. B., The anelasticity of the earth. "Journal of Geoph. Res.", 69, 10, 2071-2084, (1964).

Ben-Menahem A. and Toskoz M. N., Sonrce mechanism from spectra of long period surface waves, 2, The liamachatka Earthquake of November 4, 1952. Seism. Lab. Tech. Rep. 29, May 1963.

Bullen K. E., Rigidity and density in the earth's core. "Nature", 4921, 807, (1964).

CaLoI P., Sesse dell'Alto Adriatico con particolare riguard al Golfo di Irieste. C.N.R., R. Comitato Talassografico, Memorie, CCXLII, 1938.

CaPuto M., Elastodinamica ed elastoslatica di un modello della terra e sue auto-oscillazioni toroidali. "Boll. Geof. Teor. e Appl.", 3, 1-20, (1961).

CAPUto M., Free modes of layered oblate planets. "J. Geoph. Res." 68, 497503, (1963).

Crittenden M., Elfective viscosity of the earth derived from isostatic loading of Pleistocene Lake Bonneville. "J. Geophys. Res.", 68, 5517-5530, (1963).

Farrand W. R., Postglacial uplift in north America. "American Journal of Science", 260, 181-199, (1962).

GILBERT F., BaCkUS G., The rotational splitting of the free oscillations of the Earth 2. "Reviews in Geophysics", 1, 1-9, (1965).

KaARIAINEN E., On the recent uplift of the earth's crust in Finland. "Publ. Finnish Geodetic Institute ,12, (1953).

Knopors L., MacDonald G.J.F., Attenuation of small amplitude stress waves in solids. "Rev. of Mod. Phys.", 30, 1178.1192, (1958).

KNoporr L., Q, Reviews in Geophysics, 2, 625-660, (1964).

KowACH R. L. and ANDErson Don L., Attenuation of shear waves in the upper and lower mantle. "Bulletin of the Seismological Soc. of America", 54, 6, 1855-1864, (1964).

KrAUt E. A., Free radial modes in a compressible conducting sphere containing a uniform internal magnetic field. J. Geophys. Res. 70, 3927-3935, 1965. 
Lomnitz C., Application of the logarithmic creep law to stress wave attenuation in the solid earth. "J. Geophys. Res.", 67, 365-368, (1962).

MacDonald G. J. F., Ness N. F., A study of the free oscillations of the earth. “J. Geophys. Res.", 66, 1865-1911, (1961).

MARUSsi A., Funzione di dissipazione delle auto-oscillazioni della terra eccitate dal terremoto del Cile e registrate a Trieste. Rend. Acc. Naz. dei Lincei, Cl. Sc. Fis. Mat. e Nat., 13 Marzo 1965.

Ness N. F., Harrison J. C., Slichter L. B., Observations of the free oscillations of the earth. "J. Geophys. Res.", 66, 621-629, (1961).

Polli S., Le sesse dell'Adriatico. "Annali di Geofisica" XI, 1, (1958).

Press F., Long-period waves and free oscillations of the earth. "Research in Geophysics", 2, Solid earth and interface phenomena, M.I.T., 1964.

Press F., Seismic wave attenuation in the crust. " Journal of Geoph. Res.", 69, 20, (1964).

Slichter L. B., MacDonald G. J. F., Caputo M., Hager C. L., Report of earth tides results and of other gravity observations at Ucla. "Annales Observatoire Royal Belgique", (1964).

Slichter L. B., Seismic interpretation theory for an elastic earth. "Proc. R. Astr. Soc.", 224, 43-63, (1954).

Slichter L. B., Earth's free modes and a new gravimeter. "Geophysics", 3, 339-347, (1965).

$\mathrm{Z}_{\mathrm{ADRO}} \mathrm{M}$., "Power spectrum analysis" delle deviazioni della verticale registrate durante l'eclissi totale di sole del 15 Febbraio 1961, “Atti Convegno Ass. Geof. Ital.", X, 63-78, (1961). 